\title{
VLBI Observations of Extragalactic TeV Gamma-ray Sources
}

\author{
P.G. Edwards \\ Institute of Space and Astronautical Science, 3-1-1 Yoshinodai, \\ Sagamihara, Kanagawa 229-8510, Japan \\ B.G. Piner, S. Fodor \\ Department of Physics and Astronomy, Whittier College, 13406 E. \\ Philadelphia St., Whittier, CA 90608, USA
}

\begin{abstract}
VLBI observations of reported TeV gamma-ray sources are described and the inferences that can be drawn on the jet behaviour between the $\mathrm{TeV}$ variability-scale and the VLBI parsec-scale discussed.
\end{abstract}

\section{Introduction, Observations and Discussion}

$\mathrm{TeV}$ gamma-rays are detected using the ground-based Cherenkov technique (see, e.g., the review by Catanese and Weekes 1999). Only low redshift $(z<0.2)$ sources are expected to be detected at $\mathrm{TeV}$ energies due to absorption by the infra-red background for more distant sources - TeV gamma-ray astronomy thus allows the intergalactic infra-red background to be studied. With the current sensitivity of $\mathrm{TeV}$ gamma ray telescopes there are two established extragalactic TeV sources: Mkn $421(z=0.031)$ and Mkn $501(z=0.034)$. Detections of four other AGN have been reported but have yet to be confirmed: $1 \mathrm{ES} 2344+514(z=0.044)$, PKS $2155-304(z=0.116)$ and $3 \mathrm{C} 66 \mathrm{~A}(z=0.444$ ? $)$ (see Catanese and Weekes 1999 and references therein) and 1ES 1959+650 $(z=0.047)$ (Nishiyama et al. 1999).

The size of the emission region implied by the hour-scale variability observed in the TeV emission from Mkn421 and Mkn501 is typically 100 times smaller than that probed by Very Long Baseline Interferometry observations. However, VLBI parsec-scale imaging provides the only direct information on the jet being ejected from the core. We are undertaking multi-epoch VLBI observations of reported $\mathrm{TeV}$ gamma-ray sources to study their milli-arcsecond (mas) scale structure and component motions, and to determine whether $\mathrm{TeV}$ activity signals the emergence of a new parsec-scale jet component. In addition, we are (together with Travis Rector, NOAO) comparing the mas-scale morphology with the arcsecond-scale structure to track the jets on the larger scale (Edwards et al. 2000a).

Combination of multi-epoch VLBI observations of Mkn 421 and Mkn 501 have revealed that the jet components in these sources display sub-luminal apparent motions (Piner et al. 1999; Edwards et al. 2000b) in contrast to GeV 
gamma-ray (EGRET detected) sources, which tend to show more super-luminal motions than AGN with similar radio properties (Kellermann et al. 2000).

We have undertaken multi-epoch observations with the Very Long Baseline Array of 1ES 2344+514, PKS 2155-304 and 1ES 1959+650, and preliminary results indicate the jet components are either sub-luminal or marginally superluminal (Piner et al. 2000; Edwards et al. 2000a; Edwards et al. 2000c). 3C 66A has not been included in our observing program to date. The often quoted value for the redshift of $3 \mathrm{C} 66 \mathrm{a}$ of 0.444 is uncertain (Hewitt \& Burbidge 1993). VLBI observations by Marscher \& Marchenko (1997) revealed apparent speeds, assuming a redshift of 0.444 , of 12 to $16 c$ within 1 mas of the core and $5.6 c$ beyond 2 mas $\left(H_{0}=65 \mathrm{~km} \mathrm{~s}^{-1} \mathrm{Mpc}^{-1}\right)$. However, should the redshift be confirmed, the claimed detection of $\mathrm{TeV}$ gamma-rays must be considered suspect: conversely, should $\mathrm{TeV}$ emission be confirmed, the redshift of 0.444 is unlikely to be correct and thus these apparent speeds will need to be revised.

Despite Doppler factors of greater than $\sim 10$ inferred from short-timescale $\mathrm{TeV}$ variability and correlated $\mathrm{TeV}$ and $\mathrm{X}$-ray emission (Catanese and Weekes 1999), on the parsec-scale TeV blazars appear to show much slower motions than EGRET-detected sources.

A small angle to the line-of-sight of the jets appears unlikely. An intriguing possibility is that most of the energy and momentum is lost close to the base of the jet, where the X-ray and TeV emission occurs (Marscher 1999). As protons do not lose energy as quickly, if the deceleration of $\mathrm{TeV}$ blazar jets is confirmed, it will be a strong argument in favour of electron-positron jets in these sources.

\section{References}

Catanese, M. \& Weekes, T. 1999, PASP, 111, 1193

Edwards, P. G. et al. 2000a, in High Energy Gamma-Ray Astronomy, eds. H. J. Voelk and F. Aharonian, AIP Conf. Proc. (New York: AIP) in press

Edwards, P. G. et al. 2000b, in Astrophysical Phenomena Revealed by Space VLBI, ed. H. Hirabayashi, P. G. Edwards \& D. W. Murphy, (Sagamihara: ISAS) 235

Edwards, P. G. et al. 2000c, in Proceedings of the 5th EVN Symposium, ed. J. Conway, A. Polatidis \& R. Booth, in press

Hewitt, A. \& Burbidge, G. 1993, ApJS, 87, 451

Kellermann, K. I. et al., 2000, in Astrophysical Phenomena Revealed by Space VLBI, ed. H. Hirabayashi, P. G. Edwards \& D. W. Murphy, (Sagamihara: ISAS) 159

Marscher, A. P. 1999, Astropart. Phys., 11, 19

Marscher, A. P. \& Marchenko, S. G. 1997, in Multifrequency Monitoring of Blazars, ed. G. Tosti \& L. Takalo, (Publ. Astron. Univ. di Perugia) 68

Nishiyama, T. et al. 1999, Proc. 26th ICRC (Salt Lake City) 3, 370

Piner, B.G. et al. 1999, ApJ, 525, 176

Piner, B.G. et al. 2000, in Blazar Demographics and Physics, ASP Conf. Series, (San Francisco: ASP) in press 